\title{
Enquêter sur l'amour auprès de trois générations
}

\author{
Retour sur le rôle central des émotions dans le \\ processus de recherche qualitative
}

Caroline Henchoz ${ }^{1}$

\begin{abstract}
[Résumé] Cet article revient sur les dimensions subjectives de l'expérience de recherche d'étudiants en sciences sociales enquêtant sur la "carrière amoureuse » d'hommes et de femmes âgés de 23 à 88 ans. Il explique comment et pourquoi les émotions suscitées par les interactions entre enquêtés et enquêteurs ont acquis le statut d'outils d'investigation et se sont révélées des compétences centrales dans le processus de recherche. Leur mobilisation, leur identification et leur explicitation ont ainsi permis de rendre compte des conditions historiques et sociales de possibilité et d'impossibilité de l'expression de l'amour. Intégrer les émotions dans le processus de recherche conduit à une réorientation épistémologique: plus que les données récoltées, c'est désormais l'expérience de recherche qui devient le matériau dans lequel s'enracine la production du savoir.
\end{abstract}

Mots-clés : sentiment, émotion, méthodologie, pédagogie, entretien.

[Abstract] This article returns to the subjective dimensions of research experience by social science students investigating the love life of men and women aged between 23 to 88. It explains how and why emotions aroused by interactions between subjects and investigators have acquired the status of investigation tools and have proved to be central competencies within the research process. Mobilising, identifying and explaining these emotions thus made it possible to gain insight into the historical and social conditions relating to the possibility and impossibility of expressing love. Integrating emotions within the research process leads to an epistemological reorientation: more than the data that are collected, it is now the research experience that becomes the material on which the production of knowledge takes root.

Keywords: feeling, emotion, methodology, pedagogy, interview.

\section{Introduction}

Lors d'un séminaire de recherche que j'ai animé en 2013-2014 à l'Université de Fribourg (Suisse), vingt étudiantes et étudiants en sciences sociales ont exploré les dimensions culturelles et historiques de l'amour en travaillant sur les expériences et les

1 Université de Fribourg, Suisse département des Sciences sociales. 
perceptions de l'amour auprès de trois générations de couples mariés ${ }^{2}$. Au total, vingttrois femmes et treize hommes de 23 à 88 ans ont été interviewés par le biais d'entretiens biographiques de type compréhensif. Le choix de personnes mariées ou l'ayant été se justifiait par le taux élevé et relativement stable de mariages en Suisse durant la période couverte (Flaugergues de, 2009) et par le fait que l'amour est pour ces générations ce qui légitime l'entrée dans le mariage et sa pérennité (Kaufmann, 1993 ; Roussel, 1989).

Inspirés par plusieurs recherches portant sur sa dimension sociale et culturelle (Beall, Sternberg, 1995 ; Kruithof, 1979), nous partions de l'hypothèse que l'amour est défini et expérimenté différemment selon les générations. Les historiens (dont Ariès, Duby, 1991) ont ainsi montré que c'est à partir du XII siècle que l'amour, autrefois perçu comme instrument de désordre et de perturbation de la reproduction sociale, se transforme, avec l'amour courtois, en un élément positif sur la scène sociale. Ce n'est pourtant qu'au début du XXe siècle que l'amour dans le mariage devient la règle. Cependant, ce qu'il recouvre évolue au fil du temps. Roussel (1989) souligne ainsi qu'à partir des années 1960, la valeur de stabilité des rapports de couple est progressivement remplacée par celle de la qualité contribuant ainsi à renforcer le rôle grandissant du sentiment amoureux. Notre recherche visait à mieux comprendre ce que cela impliquait en termes de relations et de vécus amoureux.

Cet article retrace le cheminement de l'enquête et les questionnements qui ont émergés. Il explique pourquoi et comment nous en sommes venus à mobiliser les émotions suscitées par les interactions entre enquêtés et enquêteurs pour produire de la connaissance scientifique. Dans la littérature en sciences sociales, les termes « émotion" et "sentiment» sont parfois utilisés de façon interchangeable (Hochschild, 2003b). Nous retenons ici que les émotions relèvent d'un ressenti susceptible de marquer les corps (rougeur, crispation, etc.) (Petit, 2015: 12). C'est pourquoi dans ce texte, « émotion » et " ressenti » sont utilisés comme des synonymes. Hochschild (2003b : 21) décrit ainsi les émotions «comme étant le fruit d'une coopération entre le corps et une image, une pensée ou un souvenir -, une coopération dont l'individu est conscient ». Le terme "sentiment » sera quant à lui réservé à l'amour, car comme le souligne Mauss (1968 [1921]), les sentiments sont des "phénomènes sociaux». Or dans cette recherche, l'amour est défini comme un phénomène social à trois dimensions régies chacune par des règles et des conventions propres.

2 Je remercie Bugajewska Dorota, Chardonnens Tatjana, Damian Diana, Garbiec Océane, Gogniat Chris, Guerdat Ophélie, Gumy Pierre, Heiniger Mona, Koch Christina, Michel Nadège, Perdrisat Morgane, Pfulg Marina, Pinard Amandine, Reymond Kevin, Schenk Florence, Spicher Marc, Theytaz Emilie, Vallois Lucas, Yerly Gregoire, Wyssa Pauline qui ont contribué à ce texte par leurs entretiens, (r)apports et analyses. Pour des raisons de confidentialité, les prénoms ont été modifiés dans la suite du texte. Nos répondants les plus âgés se sont mariés dans les années 1970, les « pivots » dans les années 1990 et les plus jeunes vers 2000. 


\section{Définir l'amour}

L'amour romantique est, contrairement à la pratique scientifique ordinaire, rarement défini, comme s'il restait mystérieux et irrationnel (Jackson, 1991). Certains ont néanmoins tenté de le circonscrire. Ainsi Fromm (1949), Luhmann (1990 [1982]) et Cancian (1986) font de l'amour un code de conduite et de communication. Kruithof (1979) le considère comme une activité et Kaufmann (2009) un travail. Pour Boltanski (1990), c'est un mode de justification des pratiques et pour de Singly (1996), un mode de reconnaissance. Pour tenter de synthétiser ces différentes définitions, j'ai proposé dans un précédent article une définition de l'amour en tant que production ayant lieu à trois niveaux (Henchoz, 2014). Au plan institutionnel, l'amour se présente comme une idéologie, soit un patrimoine culturel commun regroupant des croyances, des représentations et des valeurs. L'industrie hollywoodienne a par exemple contribué à propager une certaine conception de l'amour romantique (Cancian, Gordon, 1988), comme le fait que la sexualité doit avoir lieu dans la relation ou que tomber amoureux n'est pas un choix rationnel. Au plan interactionnel, cette idéologie fonctionne comme une boite à outils (Swidler, 1986, 2001), c'est-à-dire comme un ensemble limité de ressources et de conventions qui fournissent des codes de conduite et des clés d'interprétation. Au plan individuel, l'amour consiste en la gestion et la production de sentiments (les siens et ceux des autres) considérés comme adéquats en regard de la situation (Hochschild, 2003b). Ces trois plans sont intrinsèquement liés. La capacité individuelle à produire les comportements attendus est centrale pour l'avenir de la relation amoureuse (Kaufmann, 2002) ou pour la reconnaissance de cette relation par autrui (Clair, 2008). Or produire un discours ou des actions qui puissent être reconnus comme de l'amour, ou «attestables» au sens de l'accountability de Garfinkel (1998 [1967]), et par conséquent capables de susciter des sentiments nécessite que les partenaires de l'échange disposent d'un stock partagé de croyances, d'images et de valeurs sur ce qu'est l'amour.

\section{2. (E)perdus d'amour}

Alors que je l'envisageais initialement pour les partenaires de la relation amoureuse, la question de l'accountability s'est rapidement imposée dans le cadre notre étude. Dès les premiers entretiens, les étudiants ont fait part de leurs difficultés à enquêter sur l'amour auprès des 45 ans et plus. À propos de sa rencontre avec Lila (74 ans), Cléa écrit dans son journal de bord : " Je lui posais des questions sur l'amour, mais elle dérivait toujours sur ses deux sujets favoris : l'argent, les différences de classe ou de générations. » Eva confirme : « J'ai insisté sur certaines questions, Cynthia [83 ans] ne les entendait pas et changeait de sujet [...].» Ce constat est également partagé par Hugues : "J'ai beau l'interroger sur l'amour, Anna [ 47 ans] n'aborde jamais le sujet. » Les étudiants estimaient ne pas recueillir les informations qu'ils sollicitaient et lorsque nous avons discuté de la manière dont se sont déroulés leurs entretiens avec les 
plus âgés, les qualificatifs les plus fréquemment mobilisés pour les décrire étaient « frustration » et « gêne ».

\section{Prendre au sérieux les émotions}

Ces qualificatifs m'ont surpris. Le guide n'était-il pas conçu de manière à susciter le récit? Du premier baiser à la période actuelle en passant par la mise en ménage et l'arrivée des enfants, les étudiants cherchaient à dessiner l'évolution de ce que nous avons appelé - en l'honneur de Becker $(1963,1985)$ et parce que cela permettait de mettre en évidence le fait que l'amour relevait d'un apprentissage - «la carrière amoureuse » des personnes rencontrées. Longs d'une heure à deux heures trente, les entretiens semblaient susciter le récit. En outre, les étudiants relevaient que leurs interlocuteurs « bavardaient » et étaient « heureux de parler ».

C'est à partir de cette étape de la recherche que nous avons intégré, dans un premier temps sans questionnement ni attente particulière, la question des émotions dans le processus de recherche. Tenter d'expliquer le ressenti des étudiants nous a pourtant conduits à progressivement changer d'orientation épistémologique. Désormais, ce n'était plus les données récoltées qui allaient nous amener à produire de la connaissance. C'était l'expérience de recherche, dans ce qu'elle faisait résonner chez les enquêteurs, qui devenait outil de production du savoir. Si historiquement les ethnologues ont fait preuve de plus de sensibilité à la démarche réflexive que les sociologues, l'intérêt des sciences sociales francophones pour les relations d'enquête et leur rôle dans la production scientifique date surtout des années 2000 (Monjaret \& Pugeault, 2014 : 6-9). Pour autant, la place du ressenti du chercheur dans ce processus reste encore peu traitée (Chauvier, 2011). Or, si l'on se base sur notre expérience de recherche, l'identification et l'intégration des émotions peuvent se révéler bénéfiques pour l'investigation en sciences sociales. Davantage que de bénéfices, nous pouvons parler de véritables compétences. Au sens de Le Boterf (1994), la compétence, ici la compétence émotionnelle, n'existe en effet pas préalablement au processus de recherche. Ce n'est pas une connaissance ou une capacité possédée que l'on mobiliserait en temps voulu. Elle ne relève pas de l'application de ressources, mais de la construction même de ces ressources. Autrement dit, c'est du « savoir mobiliser » les émotions dont il est question ici. La suite de cet article tente de montrer que les émotions suscitées par le processus d'enquête sont loin de n'être que les corollaires un peu encombrants (ou à ignorer) de la recherche qualitative. Pour peu qu'on les prenne en considération, les dimensions subjectives de l'enquête peuvent en être l'atout. En effet, elles constituent un gisement de ressources susceptibles d'être mobilisées afin de favoriser la production de nouvelles connaissances scientifiques.

\section{Aux sources des émotions}

Si la première étape a consisté à favoriser les échanges collectifs et à retourner dans les notes de terrain pour identifier et nommer les ressentis éprouvés, la seconde a visé 
à définir les sources de ces émotions. Ainsi, la «frustration » des étudiants provenait du fait que les enquêtés ne fournissaient pas le discours attendu. Lors de son entretien avec Anna, Hugues s'est par exemple montré particulièrement pugnace. Il l'a interpellée sept fois sur sa conception de l'amour idéal. Bien que celle-ci lui réponde (longuement) à chaque fois, il relance, car, comme il l'a dit plus haut, il considérait qu'elle ne lui répondait pas.

La « gêne » éprouvée par les étudiants provenait quant à elle du fait qu'ils estimaient le sujet très intime, voire intrusif. Après discussion, cette perception s'est avérée être moins rattachée aux questions posées - par exemple, « pouvez-vous me raconter votre premier amour ? » ou « qu'avez-vous ressenti lorsque vous avez rencontré la première fois celui/celle qui allait devenir votre époux/épouse ?» - qu'aux réponses obtenues. En effet, chez les plus âgés, celles-ci abordaient parfois des thématiques très personnelles comme la sexualité. Par exemple, en réponse à Malou qui le questionne sur l'évolution de ses sentiments pour sa femme, Max (88 ans) répond: "Moi, je trouve que ce n'était pas trop mal, mais tu vois quoi... à l'époque, figure-toi, pas de préservatifs, pas de pilule, hein ? Il fallait y passer !»

\section{Comprendre les émotions...}

La troisième étape de nos investigations a consisté à tenter d'expliquer ces émotions. Pour paraphraser Bourdieu (1993) : pourquoi la majorité des étudiants ressentaient-ils ce qu'ils ressentaient et pensaient-ils ce qu'ils pensaient?

Hugues nous a dit qu'il escomptait un discours sur le sentiment amoureux. Or dans ses réponses, jamais Anna ne mentionne cette dimension. Ce n'est qu'à la septième relance qu'elle aborde enfin le registre qu'il attend (mais pour dire qu'elle n'y adhère pas) : «Je ne crois pas que j'étais dans cet esprit romantique : vivre la grande passion et tout ça, non, je ne crois pas ça. »Cela met en évidence un autre point sur lequel nous reviendrons et qui relève des compétences des interviewés à pointer ce que nous pouvons appeler une "dissonance communicationnelle» (Chauvier, 2011: 67). Cette dissonance n'est pas inhérente au fait que les plus âgés ne parlent pas ou refusent de le faire, mais bien au fait qu'ils ne fournissent pas le discours permettant d'associer leurs propos à de l'amour romantique.

Sa vision de l'amour était extrêmement intéressante et différente des autres personnes [plus jeunes] interviewées. Boris [87 ans] opère dans une langue différente pour décrire l'amour et dans son récit, l'idéologie amoureuse n'existe presque pas : il se souvient peu de son mariage ; dans son appartement, il n'y a même pas de photo de lui et de sa femme, juste les photos de ses petits-enfants et arrières petits-enfants. II a utilisé le mot «naturel» pour décrire presque toutes les étapes de son histoire (le mariage était naturel, l'arrivée des enfants, la répartition des tâches, etc.). (Journal de bord de Ada)

Cet extrait relève l'absence d'indicateur de ce qui devrait constituer pour Ada l'expression appropriée de l'amour: des souvenirs clairs et précis de ce qui devrait être une étape importante de la carrière amoureuse : le mariage ; des indices matériels de cet amour comme des photos; et un discours qui devrait utiliser le langage des 
sentiments, du désir et de l'élection plutôt que celui de l'ordinaire, de la normalité et des habitudes. Cette absence d'indices suscite chez les enquêteurs de la frustration et, comme en rend compte le journal de bord de Joëlle relatant son entretien avec Adèle (25 ans), la catégorisation de la relation des plus âgés en termes de « non amour ».

C'est une histoire [...] radicalement différente de celle de ma première intervenante [Colette, 75 ans]. II s'agit ici d'une réelle histoire d'amour. On sent durant l'entretien qu'elle est très amoureuse de son mari et que les sentiments sont intensément partagés.

\section{6. ... pour rompre avec l'allant de soi}

Tenter d'expliquer les émotions ressenties par les étudiants a favorisé une rupture avec le sentiment de familiarité éprouvé face à un thème aussi ordinaire que l'amour. Les enquêtés ne fournissant pas un discours sur les émotions et les sentiments, la question s'est en effet alors posée de savoir si l'on pouvait pour autant en conclure qu'ils ne parlaient pas d'amour.

Marthe:

Robert (63 ans):
Alors, on va commencer avec les représentations de l'amour, [...] est-ce que tu peux me raconter comment tu voyais la femme idéale?

Oui, bon... Je suis né à l'époque où l'homme et la femme étaient bien distincts, dans un schéma un petit peu vieille école, aujourd'hui. Alors... I'homme était plutôt pensé pour nourrir la famille, aller travailler. La femme, plutôt pour s'occuper des enfants, évidemment rester à la maison, regarder pour le bien de la famille.

Comme le relèvent déjà Kellerhals et Cardia-Vonèche (1984) dans les années 1980 dans une enquête comparant le modèle culturel de l'amour diffusé dans les romans-photos avec les propos de couples mariés dans la moitié des années 1970, les personnes interrogées ne décrivent pas leur relation amoureuse en termes littéraires, soit comme des individus abstraits et universels, avant tout centrés sur une relation hors des contraintes du monde externe. Ils n'évoquent pas une relation soumise à des sentiments incontrôlés et focalisés uniquement sur la recherche du bonheur. À l'image des plus âgés que nous avons rencontrés, ils dépeignent un amour matériel, pragmatique, ancré sur les échanges et les productions conjugales. Cette narration de l'amour se rapproche de ce que Cancian (1986) nomme la dimension instrumentale de l'amour. Celle-ci constituerait un mode d'expression de l'amour beaucoup moins valorisé dans nos sociétés occidentales que celui qui se fonde sur une sémantique rattachée aux sentiments. En effet, ces représentations de l'amour centrées sur l'aide pratique, le partage d'activités physiques et de temps, ont durant le $\mathrm{XX}^{\mathrm{e}}$ siècle progressivement été remplacées par l'idéal du développement de soi, de la communication des sentiments, de l'intimité et des rôles de genre flexibles (Cancian, Gordon, 1988). 


\section{Le generational gap}

A l'instar du gender gap de Hochschild (2003a), nous pouvons faire l'hypothèse que le ressenti des étudiants provient d'un generational gap. Ce décalage générationnel s'expliquerait par le fait que les étudiants, dont la majorité d'entre eux a moins de 30 ans, ne partagent pas la même conception de l'amour que les plus âgés de leurs interlocuteurs. C'est à dessein que je parle surtout des étudiants. Une analyse attentive des récits collectés montre que les interviewés en semblent davantage conscients. Lorsqu'ils produisent un discours en rupture avec les attentes des enquêteurs, ils mentionnent l'écart générationnel, ce qu'ont d'ailleurs noté certains étudiants comme Cléa : «Elle parlait comme si je ne pouvais pas comprendre, car elle était comme elle disait : "d’une autre génération". »

Cette référence générationnelle peut être analysée comme une stratégie discursive mise en place par les personnes interrogées pour indiquer qu'elles font part d'une expérience qui n'est pas partagée et ainsi tenter d'expliquer ou de justifier les dissonances communicationnelles. Quand Malou demande à Max de revenir sur l'évolution de ses sentiments pour son épouse et qu'il parle de moyens de contraception, il semble conscient de produire un discours déconcertant, car il reprend aussitôt : «Il n’y a pas de comparaison, hein ? Quand tu vois les jeunes maintenant... ». Ce faisant, il restaure une certaine familiarité avec son interlocutrice et contribue ainsi à favoriser la poursuite de l'entretien. En ce sens, mettre l'accent sur les émotions du chercheur conduit indirectement à restituer le rôle central des enquêtés dans la constitution du savoir (Chauvier, 2011). Ils ont su décrypter les réactions de leurs jeunes interlocuteurs et proposer une explication (la différence générationnelle) lorsqu'ils percevaient des signes d'incompréhension. Dans cette recherche, ce sont autant les données récoltées que le processus de récolte des données en lui-même qui se sont avérés source d’informations.

\section{Recréer la familiarité}

Si l'on reprend notre définition de l'amour, ces dissonances communicationnelles entre enquêtés et enquêteurs sont liées au fait que le discours des plus âgés sur l'amour n'est pas ou peu intelligible (accountable). L'étape suivante de l'investigation est donc d'en comprendre les raisons. Autrement dit, il s'agit de questionner les conditions historiques et sociales de possibilité et d'impossibilité de l'expression de l'amour pour en quelque sorte constituer un stock commun de connaissances de manière à les rendre intelligibles. Trois pistes ont été développées. Les deux premières concernent la dimension institutionnelle. Selon leur appartenance générationnelle, les individus n’ont pas les mêmes références et les mêmes représentations rattachées à l'amour, car ils sont inscrits dans une temporalité historique et biographique différente. Comme nous l'avons vu, cela a des conséquences au niveau interactionnel, car alors ils ne disposent pas de la même boite à outils pour faire part d'une expérience que pourtant ils désignent par le même terme. Alors que ces premières pistes d'analyse se réfèrent 
au contenu de l'amour, la troisième porte sur la manière différenciée dont il est exprimé.

\section{Temporalités et représentations de l'amour}

Si l'on accepte que l'amour émerge et se construit dans un contexte historique particulier qui en oriente les contours (ce qui est possible en termes d'alliance, de pratiques sexuelles ou de modes de vie par exemple) (Beall, Sternberg, 1995), nous pouvons faire l'hypothèse que les pratiques et représentations de l'amour des plus âgés se réfèrent à des conditions sociales et historiques spécifiques. En ce sens, s'il y a dissonances communicationnelles, c'est peut-être parce qu'il manque aux étudiants des «connaissances d'arrière-plan ", c'est-à-dire un "ensemble d'informations antérieures à la conversation" aidant à la compréhension des propos des personnes interrogées (Chauvier, 2011: 62) et qu'il s'agit alors de constituer. Pour ce faire, nous avons suivi deux voies. La première a consisté à exploiter l'avantage dont bénéficiaient les personnes enquêtées. En effet, contrairement aux enquêteurs, elles avaient à disposition les connaissances d'arrière-plan qui leur manquaient. En soulignant un certain nombre de décalages générationnels, elles ont plusieurs fois fourni des indices particulièrement utiles pour reconstituer le contexte de l'époque. La seconde voie a consisté à nous pencher sur la sémantique de l'amour qui, dans les entretiens, semble relever d'une double temporalité: une temporalité historique et une temporalité biographique.

\section{La temporalité historique}

Comme nous l'avons vu plus haut dans l'échange entre Marthe et Robert, la dimension instrumentale de l'amour mise en avant par les plus âgés se réfère essentiellement à l'accomplissement de fonctions conjugales sexuées. Un détour par le contexte dans lequel s'est constituée la relation amoureuse permet de fournir quelques explications. Avant les années 1980, les divorces (OFS, 2016a) et les possibilités d'expérimenter différentes relations hors mariage sont rares. En Suisse, plus de $70 \%$ des femmes nées entre 1945 et 49 se sont mariées sans cohabitation préalable (OFS, 2016b). Cette étroite interconnexion entre amour, conjugalité et mariage explique peut-être pourquoi l'amour s'exprime en partie dans les qualités attribuées au (futur) époux. La répartition fortement sexuée du travail et la complémentarité des rôles conjugaux conduisant à une forme d'interdépendance 3 , ces attentes sont surtout ratta-

3 Selon l'Office fédéral de la statistique suisse $(2005,50)$, « en 1970, les trois quarts environ des couples avec des enfants de moins de sept ans vivaient selon le modèle du père travaillant à plein temps et de la mère au foyer ». Dans l'ancien droit matrimonial d'avant 1988, le mari, 
chées à la capacité des uns et des autres à prendre en charge les fonctions qui sont attribuées à leur sexe : gagne-pain pour Monsieur, pourvoyeuse de care pour Madame. Cette conception de l'amour semble dominante chez les hommes les plus âgés. Chez les femmes, elle côtoie une autre représentation.

À une époque où il est rarement admis de vivre seules (OFS, 2016c), les femmes quittent souvent leurs parents pour leur mari. Cela explique peut-être pourquoi l'amour est, pour un certain nombre d'entre elles, associé à l'autonomie et à l'entrée dans la vie adulte. L'amoureux idéal s'apparente à un Pygmalion émancipateur (Singly de, 1996) qui va permettre aux femmes de quitter le carcan familial par le mariage. Ainsi quand Hugues demande à Anna (47 ans) si elle avait un idéal amoureux quand elle était jeune et si elle rêvait d'une histoire d'amour, celle-ci lui répond : "Non absolument pas. Non. Je rêvais de liberté. » Interrogée sur le même sujet, Nina (57 ans) confirme: «L'homme idéal... Pas forcément... Celui qui me permettrait de m’échapper de la maison, voilà !»

Dans un tel contexte historique, on peut faire l'hypothèse que la capacité que l'on accorde à l'autre d'accomplir les fonctions de genre qui lui sont socialement attribuées (pourvoyeur de soins/de revenus ou émancipateur) contribue à susciter le sentiment amoureux. Autrement dit dans les récits, l'expression de l'amour traduit surtout l’interdépendance des époux.

\section{La temporalité biographique}

Les dissonances communicationnelles recensées peuvent aussi s'expliquer par une autre forme de temporalité : la temporalité biographique. Cette dernière est directement rattachée à la notion de carrière amoureuse. Elle se réfère au fait que l'appréhension de l'amour des personnes interrogées est chargée de leur expérience (Pagès, 2008; Schurmans, Dominicé, 1997). Selon cette seconde piste explicative, le ressenti des étudiants provient d'un décalage expérientiel. Contrairement aux personnes interrogées, ils ont rarement vécu plusieurs années avec un ou une même partenaire. Par conséquent, on peut supposer que leur conception de l'amour s'est surtout construite en référence aux normes et aux valeurs socialement dominantes alors que celle des plus âgés, comme les propos de Marie (50 ans) le soulignent, s'enracine dans l'expérience concrète de la vie quotidienne. "L'amour mature » (Pagès, 2008) décrit ici fait référence à ce qui «fonde la vie à deux », soit une "culture commune » sédimentée dans les habitudes (Kaufmann, 2009).

Au début tout feu, tout flamme comme on dit... et puis après on va plus dans ce

que j'appellerais le raisonnable [elle insiste sur le mot], le concret quoi. Heureuse-

« chef de l'union conjugale » décide de la destinée du ménage et en gère les finances alors que la femme dirige son ménage. 
ment qu'au début, on est moins dans le concret, dans le quotidien. On a plein d'idées, tout est beau et puis après, ben voilà... je ne veux pas dire qu'on redescend sur terre, mais quelque part, oui.

\section{Dire l'amour}

Enfin, il existe une troisième piste d'explication qui ne met pas l'accent sur le contenu comme précédemment, mais sur le médium : le discours. C'est l'outil de transmission mobilisé dans les entretiens, la parole, dont il est question ici. On peut en effet faire l'hypothèse que la relative absence de narration sentimentale dans les propos tenus par les plus âgés s'explique par le fait que les compétences à exprimer les émotions ne sont pas pareillement distribuées entre les générations. On sait depuis Mauss (1968 [1921]) que l'expression publique des sentiments suit des règles partagées socialement. Or la culture publique du sentiment est apparue récemment. Selon Coontz (2005), elle est à rattacher à la montée de l'individualisme qui s'est traduit par la mise en avant de l'individu et par conséquent de ses sentiments et de ses (in)satisfactions. D’autres (Cancian, Gordon, 1988; Jamieson, 1999) soulignent l'influence des mouvements politiques vers plus d'égalité entre les sexes. Ils ont conduit à changer les normes concernant l'expression des sentiments et à renforcer leur diffusion sur la scène privée et publique. La recherche de ce que l'on ressent, l'épanouissement personnel et l'authenticité s'expriment désormais publiquement et cela semble évident pour les étudiants qui, comme Giddens (2004 [1992]), définissent le romantisme contemporain par une narration dans laquelle les conjoints se racontent et racontent leur histoire. À l'instar de Jaggar (1989), nous pouvons supposer que les plus âgés sont moins socialisés à cette forme d'expression publique des sentiments et que, par conséquent, ils sont moins expérimentés pour identifier et relater ce qu'ils ressentent dans la mise en récit de soi et de ses émotions qu'exige ce mode de récolte des données spécifique qu'est l'entretien. Cela semble être tout particulièrement le cas des hommes et des classes populaires (Cancian, 1986; Pagès, 2008). Cette dernière précision permet peut-être d'expliquer pourquoi les étudiants considéraient certaines sous-populations comme étant encore moins « aimantes » que les autres.

\section{Conclusion. Le rôle des émotions dans la recherche qualitative}

Prendre au sérieux la part subjective de la recherche a permis de mettre à jour les dissonances entre enquêtés et enquêteurs par rapport à un sentiment aussi ordinaire que l'amour. Ce dialogue entre deux générations incité par la méthodologie de la recherche privilégiée, l'entretien compréhensif, a permis de rendre étrange ce qui semblait aller de soi. Les enquêteurs ont été confrontés à des propos qu'ils n'attendaient pas et les enquêtés à des réactions qui les ont conduits à mobiliser leur expérience ordinaire du changement social pour tenter de s'expliquer en invoquant le décalage générationnel. Si nous ne savons pas ce qu'ont ressenti ces derniers suite à l'entretien, faute de les avoir recontactés après, les étudiants ont pu mettre en commun leurs 
émotions. C’est dans un premier temps parce qu'elles m’ont étonnée que je les ai incités à les explorer plutôt que par souci de rompre avec le «mythe de l'investigation dépassionnée » (Kaufmann, 2009:169).

Chercher à comprendre les émotions des enquêteurs nous a conduits à procéder à une investigation triangulaire. Nous ne nous sommes pas seulement penchés sur les données récoltées, mais aussi sur l'enquêté, l'enquêteur et leurs échanges. En inscrivant les enquêtés et les enquêteurs dans une double temporalité, en revenant sur leur rapport au dévoilement discursif des sentiments, nous avons tenté de mettre à jour les conditions historiques et sociales de possibilité et d'impossibilité de l'expression de l'amour. En confrontant leurs propres catégories à celles des enquêtés, en passant de "pourquoi les interviewés les plus âgés ne tiennent-ils pas des propos qui relèvent de l'amour » à " pourquoi ne percevons-nous pas une narration relative à l'amour dans leurs entretiens?», les étudiants ont accédé aux raisonnements et aux raisons d'aimer de leurs ainés. Ils ont été amenés à dépasser la vision hégémonique de l'amour romantique contemporain. Pour entrer dans un véritable processus de socioanalyse, cette investigation aurait pu être approfondie notamment en ce qui concerne le rapport des enquêteurs à l'objet de recherche. Cela n'a toutefois pas pu être fait dans cette étude pour des raisons de temps. En outre, le rôle central de l'expérience subjective de recherche comme mode de connaissance et des émotions comme compétences et outils d'investigation ethnographiques n'est apparu que progressivement. Des études qui intégreraient plus systématiquement la place du ressenti dans le processus de recherche restent par conséquent encore à mener.

\section{Bibliographie}

ARIES P., DUBY G. (1991), Amour et sexualité en Occident, Paris, Editions du Seuil.

ATTIAS-DONFUT C. (1997), «Le cycle d'échanges entre trois générations », Lien social et politiques, RIAC, vol. 38, p. 113-122.

Beall A. E., Sternberg R. J. (1995), «The Social Construction of Love », Journal of Social and Personal Relationships, vol. 12, n 3, p. 417-438.

BeCKER H. (1985 [1963]), Outsiders. Études de sociologie de la déviance, Paris, Editions Métailié.

Boltanski L. (1990), L'amour et la justice comme compétences, Paris, Editions Métailie.

BouRdIEU P. (1993), La misère du monde, Paris, Editions du Seuil.

Cancian F. (1986), «The Feminization of Love », Signs, vol. 11, n 4, p. 692-709.

CANCIAN F., GoRdon S. (1988), « Changing Emotion Norms in Marriage : Love and Anger in U.S. Women's Magazines since 1900 », Gender e Society, vol. 2, n 3, p. 308-342.

CHAUvier E. (2011), Anthropologie de l'ordinaire. Une conversion du regard, Toulouse, Editions Anacharsis. 
Clair I. (2008), Les jeunes et l'amour dans les cités, Paris, Editions Armand-Colin.

Coontz S. (2005), Marriage, a History: From Obedience to Intimacy, or How Love Conquered Marriage, New York, Editions Viking.

FLAUGERGUes DE A. (2009), Les comportements démographiques des familles en Suisse de 1970 à 2008, Neuchâtel, Office fédéral de la statistique.

Fromm E. (1949), Man for Himself: An Inquiry into the Psychology of Ethics, Londres, Routledge.

GARFInKEL H. (1998 [1967]), Recherches en ethnométhodologie, Paris, PUF.

GIDDENS A. (2004 [1992]), La transformation de l'intimité. Sexualité, amour et érotisme dans les sociétés modernes, Rodex, Les Editions Rouergue/Chambon.

HeNCHOZ C. (2014), «La production quotidienne de l'amour en Suisse et au Québec. Comptabilités intimes », Sociologie et sociétés, vol. XLVI, n¹, p. 17-36.

Hochschild A. R. (2003a), The Second Shift, New York, Editions Penguin Books.

Hochschild A. R. (2003b), «Travail émotionnel, règles de sentiments et structure sociale », Travailler, vol. $1, \mathrm{n}^{\circ}$ 9, p. 19-49.

JACSKON S. (1991), «Even Sociologists Fall in Love: An Exploration in the Sociology of Emotions ", Sociology, vol. 27, p. 201-220.

JAGGaR A. M. (1989), « Love and Knowledge: Emotions in Feminist Epistemology », In A. M. JAGGAR, S.R. BORDO, Gender/Body/Knowledge: Feminist Reconstructions of Being and Knowing, New Brunswick, Rutgers University Press, p. 145-171.

JAMieson L. (1999), "Intimacy Transformed? A Critical Look at the "Pure Relationship" », Sociology, vol. 33, n 3, p. 477-494.

KaufMann J.-C. (1993), Sociologie du couple, Paris, PUF.

Kaufmann J.-C. (2002), Premier Matin. Comment nait une histoire d'amour, Paris, Editions Armand Colin.

Kaufmann J.-C. (2009), L'étrange histoire de l'amour heureux, Paris, Editions Armand Colin.

Kellerhals J., CARDiA-Voneche (1984), « Rôles féminins et masculins dans la relation de couple : plusieurs images pour une même culture », Revue suisse de sociologie, vol. 10, p. 755-768.

KRUITHoF C. L. (1979), L'Amour phénomène social. Étude philsophico-sociologique d'un phénomène de la vie quotidienne, Bruxelles, Editions de l'Université de Bruxelles.

Le BOTERF G. (1994), De la compétence. Essai sur un attracteur étrange, Paris, Les Editions d'organisation. 
Luhmann N. (1990 [1982]), Amour comme passion, Paris, Editions Aubier.

Mauss M. (1968 [1921]), «L'expression obligatoire des sentiments », In M. MaUSS, Essais de sociologie, Paris, Editions de Minuit, p. 81-88.

Monjaret A., Pugeault C. (dir.) (2014), Le sexe de l'enquête. Approches sociologiques et anthropologiques, Lyon, ENS Editions.

OfFice federal De la Statistique (2016a), Divorces et divorcialité depuis 1876. En ligne, consulté le 8.8.2016. URL: http://www.bfs.admin.ch/bfs/portal/fr/index/themen/01/06/blank/key/06.html.

OfFice Federal De La Statistique (2016b) Enquête sur la famille et la fécondité - Première union et vie de couple. En ligne, consulté le 8.8.2016. URL: http://www.bfs.admin.ch/bfs/portal/fr/index/themen/01/04/blank/dos/mikroze nsus/05.html.

OFFICE FEDERAL DE LA STATISTIQUE (2016c), Les personnes vivants seules. En ligne, consulté le 8.8.2016.

URL : http://www.bfs.admin.ch/bfs/portal/fr/index/regionen/thematische_karten/glei chstellungsatlas/familien und haushaltsformen/singles.html.

OfFICE FEDERAL DE LA STATISTIQUe (2005), Enquête sur les revenus et la consommation 2003, Neuchâtel, OFS

PAges M. (2008), L'amour et ses histoires: une sociologie des récits de l'expérience amoureuse, Paris, Editions L'Harmattan.

Petit E. (2015), Economie des émotions, Paris, Editions La Découverte (« Repères »).

ROUSSEL L. (1989), La famille incertaine, Paris, Editions Odile Jacob

SChURMANS M.-N., Dominice L. (1997), Le coup de foudre amoureux. Essai de sociologie compréhensive, Paris, PUF.

Singly DE F. (1996), Le soi, le couple et la famille, Paris, Editions Pocket.

Swidler A. (1986), "Culture in Action : Symbols and Strategies », American Sociological Review, vol. 51, $\mathrm{n}^{\circ} 2$, p. 273-286.

SwidLer A. (2001), Talk of Love. How Culture Matters, Chicago/Londres, The University of Chicago Press. 